\title{
A Review of the Optimisation of Photopolymer Materials for Holographic Data Storage
}

\author{
Jinxin Guo, ${ }^{1}$ Michael R. Gleeson, ${ }^{2}$ and John T. Sheridan ${ }^{1}$ \\ ${ }^{1}$ School of Electrical, Electronic and Communications Engineering, College of Engineering and Architecture, \\ Communications and Optoelectronic Research Centre, The SFI-Strategic Research Cluster in Solar Energy Conversion, \\ University College Dublin, Belfield, Dublin 4, Ireland \\ ${ }^{2}$ Department of Computer Science, National University of Ireland Maynooth, Maynooth Co. Kildare, Ireland
}

Correspondence should be addressed to John T. Sheridan, john.sheridan@ucd.ie

Received 25 April 2012; Accepted 4 May 2012

Academic Editor: Sergi Gallego

Copyright ( $) 2012$ Jinxin Guo et al. This is an open access article distributed under the Creative Commons Attribution License, which permits unrestricted use, distribution, and reproduction in any medium, provided the original work is properly cited.

Photopolymers are very interesting as optically sensitive recording media due to the fact that they are inexpensive, self-processing materials with the ability to capture low-loss, high-fidelity volume recordings of 3D illuminating patterns. We have prepared this paper in part in order to enable the recognition of outstanding issues, which limit in particular the data storage capacity in holographic data storage media. In an attempt to further develop the data storage capacity and quality of the information stored, that is, the material sensitivity and resolution, a deeper understanding of such materials in order to improve them has become ever more crucial. In this paper a brief review of the optimisation of photopolymer materials for holographic data storage (HDS) applications is described. The key contributions of each work examined and many of the suggestions made for the improvement of the different photopolymer material discussed are presented.

\section{Introduction}

Despite improvement to a range of standard storage media, such as solid state memory/magnetic tape [1], there is an every growing need to increase the capabilities and flexibility of data storage systems. To succeed further in the data storage market, optical storage technology must compete effectively against magnetic tape on all fronts: cost, capacity, and data transfer rate [2]. One great potential optical method uses a holographic approach, where recorded data are distributed throughout the volume of a thick medium. Photopolymer materials are being actively studied for practical applications such as Holographic Data Storage (HDS), hybrid optoelectronics, photo embossing, including the manufacture of refractive and diffractive optical elements, and the self-trapping of light [3-9]. Their versatility, ease of use, and selfprocessing ability give them many advantages over more traditional materials such as silver halide and DCG $[10,11]$. The necessity for development and optimization of a photopolymer system, with higher storage capability and stability, has been recognized, and they have recently received significant attention.

In this paper, we review recent attempts to more fully understand what is needed to optimise the performance of photopolymer materials for HDS applications. Specifically we aim to discuss the evolution of our understanding of what takes place inside these materials and what happens during and after holographic recording, with the objective of further improving the performance of such materials.

The paper is structured as follows. In Section 2, we briefly review the photochemical processes generally occurring during the fabrication of refractive index structures in freeradical photopolymerisation material systems. In Section 3, a description of some typical measurements performed in order to optically characterise photopolymer materials is presented. In Section 4, we begin by briefly discussing a range of different photopolymer materials, which are currently being developed for potential use as HDS media. Then an overview of optimisation of photopolymer materials in the literature is presented. A brief conclusion is given in Section 5. 
A discussion of several issues, which limit the performance of such materials and some potential improvements, is presented.

\section{Photochemical Processes}

In typical free radical photopolymerization systems, the kinetic model describing what takes place involves five main processes: (I) initiation, (II) propagation, (III) termination, (IV) inhibition, and (V) chain transfer, each of which may involve several physicochemical reactions. We discuss the major chemical reactions taking place in each process below [12-16].

(I) Initiation. During illumination, the reaction between the photosensitiser and the electron donor (coinitiator) leads to the production of initiator radicals, $R^{\bullet}$, which can react with the monomers to produce chain initiators, $M^{\bullet}[14]$ :

$$
\begin{gathered}
I \stackrel{h v}{\rightarrow} R^{\bullet}, \\
R^{\bullet}+M \stackrel{k_{i}}{\rightarrow} M^{\bullet},
\end{gathered}
$$

where $I$ is the initiator, $h v$ indicates the energy absorbed from a photon, $k_{i}$ is the chain initiation kinetic constant, and $M$ represents a monomer molecule.

(II) Propagation. The chain initiator, $M_{1}^{\bullet}$, will attach itself to another monomer molecule, $M$, by addition to the $\mathrm{C}=\mathrm{C}$ bond yielding a growing polymer radical with an active tip. Through propagation the polymer chain grows $[11,12]$ :

$$
M_{n}^{\bullet}+M \stackrel{k_{p}}{\rightarrow} M_{n+1}^{\bullet}
$$

where $k_{p}$ is the rate constant of propagation and $M_{n}^{\bullet}$ and $M_{n+1}^{\bullet}$ are the growing macroradical chains of $n$ and $(n+1)$ repeat monomeric units $(n \geq 1)$.

(III) Termination. Termination can occur in three ways. Two of these, disproportionation and combination, involve two growing macroradicals interacting, that is, the bimolecular termination mechanism:

$$
\begin{aligned}
& M_{n}^{\bullet}+M_{m}^{\bullet} \stackrel{k_{\mathrm{tc}}}{\rightarrow} M_{n+m}, \\
& M_{n}^{\bullet}+M_{m}^{\bullet} \stackrel{k_{\mathrm{td}}}{\rightarrow} M_{n}+M_{m},
\end{aligned}
$$

where $k_{\mathrm{tc}}$ and $k_{\mathrm{td}}$ are the rate constants of combination and disproportionation termination, respectively. $M_{n}, M_{m}$, and $M_{n+m}$ represent terminated chains which have no radical tip, that is, a dead polymer chain.

A third possible termination mechanism involves primary radical termination $[14,17]$ :

$$
M_{n}^{\bullet}+R^{\bullet} \stackrel{k_{\mathrm{tp}}}{\rightarrow} M_{n} R
$$

where $k_{\mathrm{tp}}$ is the rate constant of primary radical termination. In this step, a growing macro-radical chain reacts with a primary radical (initiator radical) leading once again to the production of inactive or dead polymer chains [14].
(IV) Inhibition. Inhibitors are chemicals which react with the initiating and propagating radical species by rapidly removing or scavenging these radicals. Polymerization is completely halted until they are all consumed [13]. Several possible inhibitor reaction mechanisms are listed below:

$$
\begin{gathered}
\text { Dye }^{*}+Z \stackrel{k_{z, D}}{\rightarrow} \text { LeucoDye }+Z^{*}, \\
R^{\bullet}+Z^{\stackrel{k_{z, R^{\bullet}}}{\rightarrow}}\left(R+Z^{\bullet}, \text { and/or } R Z^{\bullet}\right), \\
M_{n}^{\bullet}+Z^{k_{z, M^{\bullet}}}\left(M_{n}+Z^{\bullet}, \text { and/or } M_{n} Z^{\bullet}\right),
\end{gathered}
$$

where $Z$ is the inhibitor species, for example, oxygen, Dye* is the excited photosensitiser, $Z^{\bullet}$ is the concentration of singlet oxygen $[13,16-18]$, and $k_{z, D}, k_{z, M} \cdot$, and $k_{z, R^{*}}$ are the rate constants of inhibition of the photosensitiser, the macroradicals, and the primary radicals, respectively. Inhibition leads to a dead band at the start exposure, that is, no initial grating formation during exposure. The effects of inhibitors are especially significant when lower exposure energies are used, for example, when large areas must be exposed or short pulses must be used [14].

(V) Chain Transfer Mechanism. In many polymerization systems, the average polymer weight is observed to be lower than predicted by the chain transfer reaction $[13,19-21]$. Generally, the chain transfer process causes the premature termination of a growing macro-radical chain and arises because of the presence of a chain transfer agent (CTA) [13]. Due to this reaction, a new radical is produced which is referred as a reinitiator. This reinitiator reacts with a monomer molecule to initiate a new growing macro-radical chain. The chain transfer reactions can be written as

$$
\begin{gathered}
M_{n}^{\bullet}+(\mathrm{RI}-\mathrm{X}) \stackrel{k_{\mathrm{tr}, s}}{\rightarrow}\left(M_{n}-\mathrm{X}\right)+\mathrm{RI}^{\bullet}, \\
\mathrm{RI}^{\bullet}+M \stackrel{k_{\mathrm{ri}}}{\rightarrow}\left(\mathrm{RI}-M^{\bullet}\right),
\end{gathered}
$$

where RI-X is the chain transfer agent, $-\mathrm{X}$ is the atom or species transferred, and $\mathrm{RI}^{\bullet}$ is the reinitiator which has a radical tip. $k_{\mathrm{tr}, S}$ and $k_{\mathrm{ri}}$ are the transfer rate constant to chain-transfer agent and the re-initiation rate constant, respectively. Due to the premature termination reaction with the chain transfer agent, RI-X, the propagating polymer chains will stop growing earlier than they would have if the CTA was not present. We assume that the free radical RI- $M^{\bullet}$ produced can be treated as acting chemically identical to a chain initiator $M^{\bullet}$. Therefore the reinitiator, $\mathrm{RI}^{\bullet}$, simply initiates a new growing chain with a radical tip $M^{\bullet}$. Thus, while the polymer chains are shortened, the amount of monomer polymerized and the rate of polymerization can remain high.

In the above we have presented the main photochemical processes which take place in general free-radical photopolymerisation materials. However, the processes may vary in complexity and significance in different photopolymer systems. Furthermore, Figure 1 illustrates simple 1D grating formation process in such a photopolymer material. It can be seen from the schematic that polymerisation occurs most 


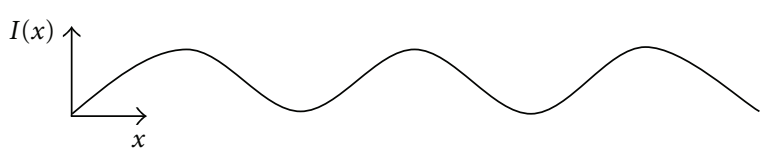

(a)

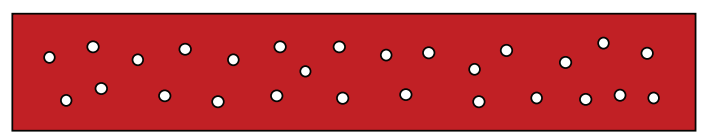

(b)

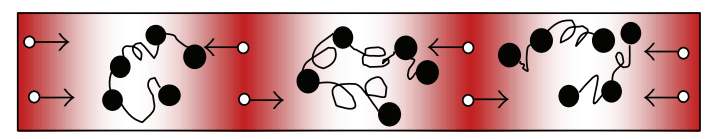

- Monomer

- Polymer

(c)

FIGURE 1: Grating formation in photopolymer where (a) represents the sinusoidal illuminating intensity distribution at the plate; (b) represents the photopolymer layer before recording; (c) represents the photopolymer layer during recording.

strongly in the bright regions of high exposure due to the sinusoidal illuminating intensity pattern. As monomer is consumed in these regions due to polymerisation, a monomer concentration gradient is created. In the weakly illuminated regions, the excess monomer diffuses into the brighter regions in order to eliminate the resulting concentration gradient. This is illustrated in Figure $1(\mathrm{c})$. As a result, a sinusoidal polymer concentration distribution is formed. Assuming all the monomer is converted to polymer by the end of the recording process, the spatial variation in the permittivity of the material will be related to the polymer concentration distribution. Thus a permanent modulation of the layer permittivity is generated; that is, a volume refractive index grating structure is produced.

\section{Holographic Setup and Measurements}

In order to optically characterise holographic recording materials, the gratings fabricated are often modeled using Kogelnik's two-wave coupled-wave theory [22]. In 1981, Moharam and Gaylord [23] presented a rigorous coupledwave approach, which included the second derivatives and boundary diffraction, to obtain a stable algorithm for thick material layers. More rigorous models have been developed, see Syms [24], based on the electromagnetic theory, in order to explore more accurately what happens in volume (thick) hologram. Comparing with the simple coupled wave theory, such models can involve the use of complex computational techniques but deal with more complicated grating structures. Kogelnik's two-wave coupled-wave theory is both simple and practical and describes the efficiency with which thick holograms can diffract incident light. Analytic expressions for both the angular and wavelength dependence of sinusoidal grating diffraction efficiency as the incident light deviates from the Bragg condition are available. Thus the dependence of the diffraction efficiency, $\eta(t)$, on a number of grating parameters is known. For a lossless, unslanted transmission geometry grating replayed on Bragg with TEpolarized probe light [22], that $\eta(t)$ is described by the following equation:

$$
\eta(t)=\frac{I_{D}(t)}{I_{\text {in }}}=\sin ^{2}\left(\frac{\pi d n_{1}(t)}{\lambda \cos \theta}\right),
$$

where $I_{\text {in }}$ and $I_{D}(t)$ are the incident and diffracted probe beam intensities, respectively, $d$ is the grating thickness, $\theta$ and $\lambda$ represent the Bragg angle and wavelength of the incident probe beam inside the grating layer, and $n_{1}(t)$ is the amplitude of the first harmonic time-dependent refractive index modulation. We note that in deriving (7), all boundary reflections have been neglected. This equation can be rearranged to give a convenient expression for the temporally varying refractive index modulation, $n_{1}(t)$, as follows:

$$
n_{1}(t)=\frac{\lambda \sin ^{-1}(\sqrt{\eta(t)}) \cos \theta}{\pi d} .
$$

By monitoring the amount of light diffracted from a weak probe beam during exposure, $I_{D}(t)$, growth curves of refractive index modulation against exposure time can be extracted. In most of the literature, reviewed below, such growth curves are used to monitor grating formation. A typical experimental setup used to obtain such growth curves is presented in Figure 2 [25].

During the recording process, the evolution of the grating in real time is monitored. One of the main advantages of many photopolymer materials is that they are self-processing and thus nonlatent [22], and therefore the diffractive scattering properties are immediately available as the grating is being formed. This allows the evolution of the grating to be monitored during exposure by replaying the grating as it is being recorded using a probe laser with a wavelength which lies outside the absorption spectrum of the photosensitizer used. This ensures that the probing does not affect the fabrication process. Using the setup as showed in Figure 2, it is possible to monitor the grating formation (growth curve) by recording the intensity of the diffracted beam, $I_{D}(t)$, to estimate $n_{1}(t)$ using $(8)$. The angular response of the grating can also be obtained by introducing a rotation stage into the setup. The resulting system allows the recording of grating arrays, and appropriate processing of the measured intensity data allows other grating parameter values to be extracted, that is, grating thickness and nonuniformities $[22,26]$.

\section{Photopolymer Materials and Optimisations}

Photopolymers generally consist of a monomer, a photosensitive dye, and an initiator. They can either be liquid or dry layer systems. The dry photopolymers usually contain a polymeric binder in addition to the other components in order to produce dry photopolymer layers. Several photopolymer materials, which have received attention during HDS media studies, are now discussed. 


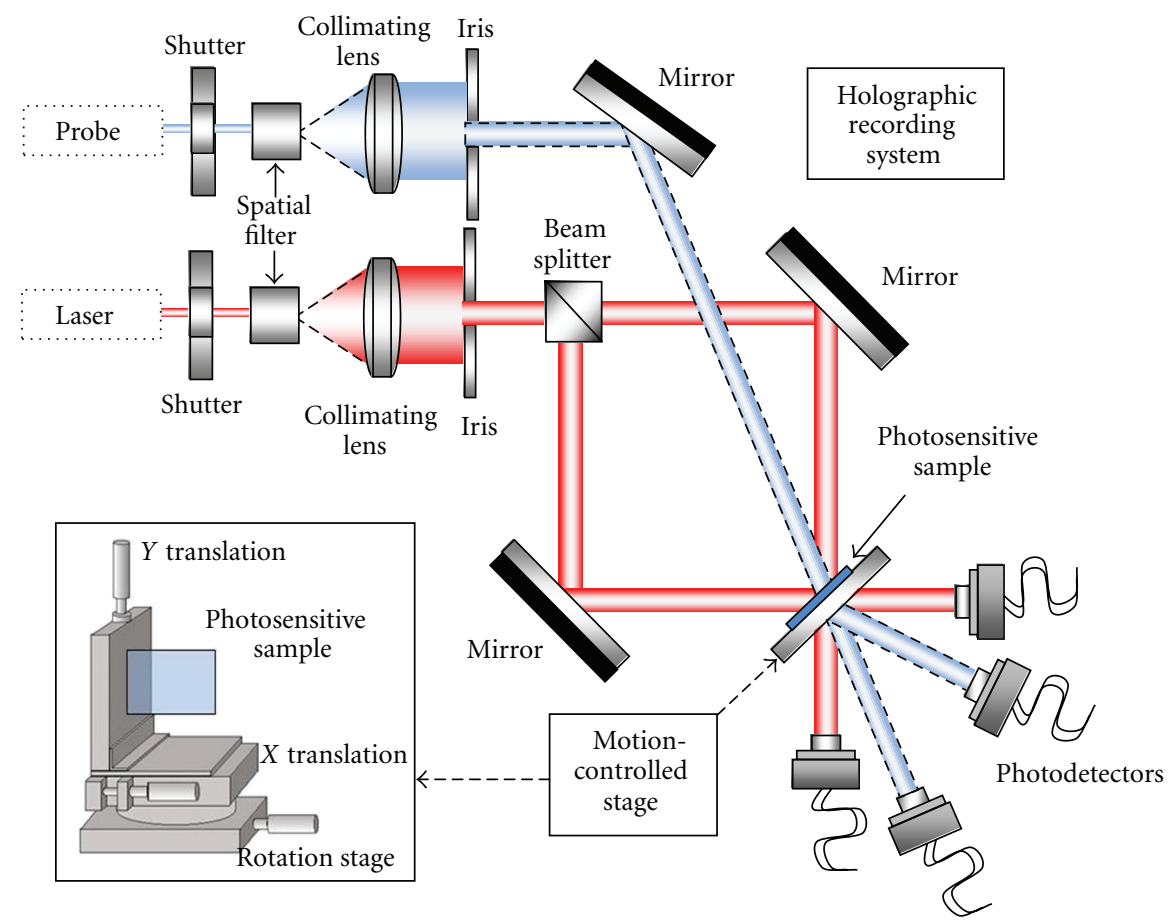

FIGURE 2: Typical experimental setup to record unslanted volume transmission holographic gratings with a recording wavelength of $\lambda=$ $633 \mathrm{~nm}$ and a probing wavelength of $\lambda=430 \mathrm{~nm}$.

Photopolymers were first introduced as a holographic recording material by Close et al. in 1969 [27]. This liquid state material consisted of a mixture of acrylamide and metal acrylate monomers and a photocatalyst, methylene blue. Sadlej and Smolinska [28] improved the original system proposed by Close by including a polyvinyl alcohol (PVA) binder which allowed the production of dry photopolymer layers. In the 1980s, Calixto [29] continued the work on acrylamidebased systems. The material contained acrylamide monomer, TEA as an electron donor, methylene blue photosensitizer, and PVA as a binder. Blaya et al. [30] improved the sensitivity of the acrylamide material for recording at 633 $\mathrm{nm}$ by changing the cross-linker, $\mathrm{N}, \mathrm{N}^{\prime}-(1,2$-Dihydroxyethylene)bisacrylamide. A hybrid material containing acrylamide and acrylic acid as monomers was proposed by Zhao et al. [31]. The material uses methylene blue as the photosensitizer, TEA and p-toluenesulfonic acid as sensitizers, and gelatin as a binder.

Trentler et al. [32] developed an epoxy resin photopolymer material with a solid matrix, which is formed in situ as the epoxy cures at room temperature. The unreacted vinyl monomers within the material are subsequently photopolymerized during hologram recording. One of the key features of this type of material is the separation of the epoxy and vinyl polymerizations. This separation allows for a large index contrast to be developed in holograms when components are optimized. This material is functional in thick formats (several millimetres), which enables narrow angular bandwidth and high diffraction efficiency. A dynamic range $(M / \#)$ up to 13 has been measured in these materials.
Eich et al. [33] introduced a type of photopolymer material with liquid crystals, which offered a completely different method for optical data storage. Following this work, various functionalized systems, with liquid crystals and/or optical chromophores, commonly referred in the literature as photoaddressable polymers (PAPs), were developed. Zilker et al. [34] improved these types of materials by introducing a new concept, donor-acceptor substitutions, which lead to an increase of the interaction between azo and mesogen groups. A refractive index modulation up to 0.2 has been obtained in these materials.

While materials above have received much attention in the literature, attempts have also been made to improve the refractive index modulation of a photopolymerizable medium by introducing diffusible high refractive index species, such as titania nanoparticles, into the regular photopolymerisable layers. The refractive index modulation in such materials is comparable to those for holographic polymer-dispersed liquid crystals [35]. This type of approach also offers one possible way to suppress the effects of polymer shrinkage, which occur due to volumetric changes during photopolymerisation process [35].

In 1998, Blaya et al. [36] investigated the effects of the addition of a cross-linking agent (N,N'-methylenebisacrylamide) to a photopolymerisable matrix for HDS. A nonlinear response of the material with regard to the storage intensity was observed. Diffraction efficiency of around $88 \%$ was obtained with energy exposure of $12 \mathrm{~mJ} / \mathrm{cm}^{2}$. At that time, the sensitivity of this material approached that of commercial films without the use of postprocessing, which was necessary 
in other materials. Later in 1998, the effects of the exposing intensity, the thickness, and the variation of the concentration of each component were experimentally examined by Blaya et al. [37]. Diffraction efficiencies of $80 \%$, with energetic sensitivities of $40 \mathrm{~mJ} / \mathrm{cm}^{2}$, were obtained in photosensitive films of a $35 \mu \mathrm{m}$ thickness at a spatial frequency of 1000 lines $/ \mathrm{mm}$.

In 1999, Mallavia et al. [38] developed a photopolymerizable system composed of a xanthene dye and N-methyldiethanolamine (MDEA) as photoinitiator with a monomer mixture of 2-hydroxyethyl metacrylate (HEMA) and pentaerythritol triacrylate (PETA). The best results reported were diffraction efficiencies over $40 \%$ with energetic exposures of $800 \mathrm{~mJ} / \mathrm{cm}^{2}$, with a decrease of inhibition time when high concentrations of PETA $(>67 \%)$ were used.

At the same time, Fimia et al. [39] reported a new aqueous photopolymer containing the monomers acrylamide, $\mathrm{N}, \mathrm{N}^{\prime}$-methylenebisacrylamide and zinc acrylate, the initiators 4,s-diiodosuccinylfluorescein (2ISF) and methylene blue $(\mathrm{MB})$, and the coinitiator sodium p-toluenesulphinate. This formulation enhanced the high-energy sensitivity at 514 or $633 \mathrm{~nm}$, compared to that of the same mixture but with only one of the two dyes. Maximum diffraction efficiencies of 15$20 \%$ for $15-60 \mathrm{~mJ} / \mathrm{cm}^{2}$ were reached, due to the combined action of the cationic and anionic dyes as visible photoinitiators. The enhancement observed, which arose due to the developer mixture in photographic emulsions, suggested that such mixture could be of potential utility when recording multifunctional diffractive systems.

In 2000, Karpov et al. [40] proposed a theoretical model for hologram recording in photoformers (polymer aggregates with polymerization diffusion mechanism of refractive index modulation and/or absorption coefficient modulation). This method is derived from the rate equations, which describe the sequence of chemical transformations in the photoformer under illumination using an appropriate wavelength and power. A set of equations which take into account the nonlinear diffusion and the multicomponent diffusion (monomer, polymer, holes) effects is presented. This model is then fitted to experimental data in an attempt to estimate the kinetics involved during photopolymerization, such as the polymer chain termination order and diffusivity.

In 2001, O'Neill et al. [41] examined the use of an aerosol sealant (varnish) to seal the photopolymer holographic recording material from the environment. Application of the sealant was found to produce some deterioration in the optical quality of the resulting holographic optical elements (HOEs). It was also shown to produce an increased lifetime of the active material (prerecording), an increase in the diffraction efficiency from the resulting diffraction gratings recorded, and improved HOE shelf life. While some of the material characteristics were improved by the introduction of the varnish layer, an increase diffuse scatter was observed during the recording layers producing. This technique was chosen to replace coverplating as it was found to be a cheaper and simpler way to seal the photopolymer recording material from the environment. The coverplating material in general leads to better humidity stability and more stable long-term diffraction efficiency.
In 2002, in work reported by Yao et al. [42], an acrylamide-based polymeric film was optimised and characterised for holographic recording. Diffraction efficiencies of 55\%, with the energetic sensitivity of $60 \mathrm{~mJ} / \mathrm{cm}^{2}$, were obtained in the photosensitive films of $150 \mu \mathrm{m}$ thickness with a spatial frequency of 2750 lines $/ \mathrm{mm}$. The paper indicated that each component had an optimal concentration and that it is not the case that the higher the concentration, the better the holographic recording characteristics. The effects of several components' concentrations were examined experimentally in this work.

In the same year, Suzuki et al. [35] demonstrated stable holographic storage in the green with high diffraction efficiency and recording sensitivity in $\mathrm{TiO}_{2}$ nanoparticle-dispersed methacrylate photopolymer films. It was shown that the diffraction efficiency as well as the recording sensitivity significantly increases with increasing nanoparticle concentration. It was also found that volumetric shrinkage during holographic exposure is noticeably suppressed by their inclusion. A fractional change of $2.9 \%$ in thickness was estimated. A refractive index modulation of $5.1 \times 10^{-3}$ with sensitivity $S=1.8 \times 10^{-3} \mathrm{~cm}^{2} / \mathrm{J}$ in this material was reported.

In 2003, Tomita and Nishibiraki [43] extended the results in [35] reporting a more than one-order-of-magnitude improvement in holographic recording sensitivities in the green by doping pyrromethene dyes into $\mathrm{SiO}_{2}$ nanoparticledispersed methacrylate photopolymer films. Holographic recording dynamics were measured for several dye concentrations and writing intensities. It was found that there exists an optimum recording intensity for a given dye concentration to maximize the strength of a permanent (fixed) volume hologram. It was shown that the doped dye concentrations lower than $0.1 \mathrm{wt} \%$ were preferable when the thickness of sample layer is $50 \mu \mathrm{m}$. The sample with $0.05 \mathrm{wt} \%$ of dye doped increased sensitivity $S$ and dynamic sensitivity $S^{*}$ that were 9 and 12 times the values, $4.2 \times 10^{-4}$ and $1.2 \times$ $10^{-4} \mathrm{~cm}^{2} / \mathrm{J}$, for undoped sample.

Later in the same year, Blaya et al. [44] also reported a photopolymerisable dry polymeric film based on pyrromethene dye. This new composition was based on three components: poly-(methylmethacrylate) (PMMA) as a binder, 2-hydroxyethylmethacrylate (HEMA) as a monomer and 1,3,5,7,8-pentamethyl-2,6-diethylpyrromethene-, difluoroborate as photoinitiator. Diffraction efficiencies near $60 \%$ were obtained with exposures of $\approx 1 \mathrm{~J} / \mathrm{cm}^{2}$ in $500 \mu \mathrm{m}$ thick layers. The properties of the material were also examined when the concentration of monomer and the exposing intensity were changed. Good results were obtained for low exposure intensities, which imply that this material could be used for low power applications.

Ortuno et al. [45] developed a method of preparing layers of a polyvinyl alcohol/acrylamide-based photopolymer approximately $1 \mathrm{~mm}$ thick. Optimization of this material made it possible to obtain diffraction efficiencies up to $70 \%$ and energetic sensitivity of $50 \mathrm{~mJ} / \mathrm{cm}^{2}$. In order to increase the final thickness of the layer, the PVA concentration in the initial solution was increased; because of this, a greater amount of water is retained by the PVA in the dry layer. 
In 2004, Cho et al. [46] reported that both the diffraction efficiency and morphology of holographic gratings were improved by combining ring-opening polymerizable epoxides with radical polymerizable conventional acrylate systems. By adding epoxide materials, the grating spacing was increased when compared with the case where only acrylate was used. The effects were especially notable for cyclohexane oxide derivatives. The diffraction efficiency was the highest, and nanoscale phase-separated liquid crystal domains were the most clearly formed using siloxane-containing cyclohexane oxide group, due to both their incompatibility with other components and fast curing. Their addition to ordinary vinyl polymerization system also reduced the volume shrinkage. However, specific values regarding the diffraction efficiencies and shrinkage obtained were not presented in this work.

In 2005, McLeod et al. [47] showed that with the addition of a reflecting head and a confocal pinhole, a standard optical drive can read and write many layers of microholographic tracks in a rapidly rotating, homogeneous holographic photopolymer disk. The analysis revealed that the index change available for each layer is the total possible change over the number of layers, analogous to results for page-based holographic multiplexing. The experiment demonstrated that photopolymer materials specifically developed for pagebased holographic storage could potentially be used in a modified traditional optical drive to achieve performance comparable with page-based holographic storage but at a reduced write/read system complexity. In comparison with page-based holographic storage, the rapid-disk rotation of the bit-based storage permits faster access time, and the total addressable storage space was shown to be as large as pagebased systems at comparable operating points. The analysis was experimentally validated at $532 \mathrm{~nm}$ by writing and reading 12 layers of microholograms in a $125 \mu \mathrm{m}$ photopolymer disk continuously rotating at $3600 \mathrm{rpm}$. The experimental results predicted a capacity limit of 140 Gbytes in a millimeter-thick disk or over 1 Tbyte in a system using the wavelength and numerical aperture of Blu-Ray technique.

Kelly et al. [48] proposed an extension to the nonlocal polymerization-driven diffusion model (NPDD) model [49, 50] to account for the temporal response associated with polymer chain growth. This was achieved by including an exponential temporal material response along with the nonlocal spatial material response function. Previously, all temporal effects were assumed to be instantaneous and were neglected. Using RCWA and the Lorentz-Lorenz expression, the temporal evolution of refractive index modulation was determined during and after illumination. Comparisons were then made between the theory and experiment for two models. In the first, the dominant termination mechanism was assumed to be bimolecular, while in the second, primary termination was assumed to be dominant. Material parameters were then extracted based on best fits to the experimental data for both models. For the two models examined, values for the diffusion coefficient were found to be of the order, $D \sim 10^{-11} \mathrm{~cm}^{2} \mathrm{~s}^{-1}$, the nonlocal spatial response parameter was found to be $\sqrt{\sigma} \approx 60 \mathrm{~nm}$, and the nonlocal temporal response parameter was found to be $\tau_{n} \sim 0.1 \mathrm{~s}$. The best fits, that is, higher correlation, to the experimental data were achieved assuming the primary termination model.

O'Neill et al. [51] examined the temporal evolution of the optically induced surface relief pattern in acrylamide-based holographic recording materials during and after exposure to intense light. In this work, they examined the effects of coherent exposure energy and mask pattern on the final surface relief pattern. The temporal evolution of the exposed spot was estimated. The $44 \mu \mathrm{m}$ thick photopolymer layer was exposed using a $24 \mathrm{~mJ} / \mathrm{cm}^{2}$ beam. It clearly showed that there is an initial shrinkage, $\sim 0.34 \mu \mathrm{m}$, followed by a period during which swelling occurs. The resulting profile was a pedestal $\sim 1.2 \mu \mathrm{m}$ in height. The effects of varying the exposure energy on the resulting temporal behavior of the central height were also examined. The height parameter was defined as the difference between the edge and the center of the exposure. The material variation increases with increasing intensity as was expected. From an examination of the effects of exposing the layer with both single-beam and double-beam (holographic) illumination, it was found that although the swelling is less for the two-beam recording, it is still appreciable. The layers examined were 47 and $50 \mu \mathrm{m}$ thick for the double-beam and single-beam exposures, respectively. The exposure energy in both cases was $\sim 240 \mathrm{~mJ} / \mathrm{cm}^{2}$. This result has implications for holographic recording in this material as shrinkage/swelling of the material can result in Bragg detuning effects, which are important for slanted gratings recording. This study also showed that it is possible to use the volume change in holographic recording materials to produce patterned surfaces.

In 2006, Wei et al. [52] presented a dual-channel holographic recording technique and its corresponding memory scheme using a cationic ring-opening photopolymer. In this dual-channel technique, a pair of holograms is recorded simultaneously with two orthogonal polarization channels in the common volume of the material and is reconstructed concurrently with negligible interchannel crosstalk. A holographic memory system with equal diffraction intensities (in each page) was developed by combining the dualchannel technique with a speckle shift multiplexing method. In addition, this system used only one $4-f$ optical head to transfer the pair of data pages with orthogonal polarization. This simultaneous use of the two channels provided a compact system with a fast data transfer rate in both the recording and reading processes.

In the same year, Tomita et al. [53] reported the use of hyperbranched polymers (HBPs), acting as mobile organic nanoparticles, dispersed in methacrylatephotopolymersfor highly efficient volume holographic recording. Two types of reduced HBPs, hyperbranched poly (ethyl methacrylate) (HPEMA) and hyperbranched polystyrene (HPS), having the average size of $10 \mathrm{~nm}$ were synthesized. This type of material provided diffraction efficiency near $100 \%$ at a recording intensity of $100 \mathrm{~mW}$ in the green $(532 \mathrm{~nm})$ recording. An approximately $60 \%$ reduction in the fractional volume change was obtained compared to the undoped material sample; that is, there was substantial polymerization-shrinkage suppression in this photopolymer system. 
John et al. [54] developed a new erasable photopolymerisable recording media based on poly (vinyl alcohol) and vinyl acetate (PVA and VAc) sensitized with methylene blue. The PVA-VAc ratio was optimised at $2: 1$. Diffraction efficiency of $6.3 \%$ was obtained without any fixing at a dye concentration of $9.3 \times 10^{-4} \mathrm{~mol} / \mathrm{L}$ and an exposure of $750 \mathrm{~mJ} /$ $\mathrm{cm}^{2}$. The material is attractive on account of its reusability. The recorded gratings were found to remain in the material for 24 hours with a continuous fall in diffraction efficiency. In order to examine the reusability of the MBPVA/VAc system, gratings were again recorded on the same area of the same film after 24 hours, that is, after the absorption spectra showed complete recovery. Their results showed that the MBPVA/VAc system could be reused 24 times. Being an inexpensive, nontoxic material with high environmental stability and good optical clarity, this material is of some interest for rewritable holographic data storage.

Fernandez et al. [55] reported a combination of two multiplexing methods, that is, peristrophic and angular multiplexing, for hologram recording in the acrylamide-based photopolymer material layers. In order to obtain holograms with higher and more uniform diffraction efficiency in thick material layers, an optimised exposure schedule method was used during the recordings. A dynamic range of $M \#=8.7$, with an angular selectivity of $0.5^{\circ}$, was obtained. Mean diffraction efficiencies of $1.8 \%$ were also obtained from 60 holograms recorded.

In 2007, in order to enhance the material's energetic sensitivity, Jeong et al. [56] introduced an epoxy-resin crosslinked matrix into an acrylamide-based photopolymer material. Diffraction efficiency near $92 \%$ and an energetic sensitivity of $11.7 \times 10^{-3} \mathrm{~cm}^{2} / J$ were achieved by the additions of epoxy-resin-based matrix. Value of volume shrinkage of $0.67 \%$ was also obtained. It was found that the epoxy-resinbased cross-link photopolymer with low cross-linking density, which could significantly enhance the energetic sensitivity without reducing both the diffraction efficiency and dimensional stability.

Ling et al. [57] used a triangle prism tilted at $13.7^{\circ}$ to the axis within the focus of a lens, in order to reduce the depth variation of recording intensity in thick $(1 \mathrm{~mm})$ polyvinyl alcohol/acrylamide (PVA/acrylamide) photopolymer layers. A mean diffraction efficiency of $9.5 \%$ was obtained for the modulated recording lights. From the results, the larger dynamic range, $M \#=9.2$, was obtained as a result of decreasing the attenuation in depth of light of the material.

Li et al. [58] demonstrated three-dimensional erasable bit optical data storage in a quantum-dot doped photopolymer under two-photon excitation using a near infrared femtosecond pulsed laser beam. It was shown that the photorefractive polymer, consisting of poly(vinyl carbazole), ethyl carbazole, 4-(dimethylaminobenzylidene)-malononitrile, and CdS quantum dots (QDs), exhibited changes not only in refractive index but also in fluorescence. It was found that, after incorporating CdS QDs with a sulfur-rich surface, the rewritable recording range of the sample was more than $200 \%$ that of the sample without QDs. Such a photosensitivity provided a multimode readout mechanism, which could be potentially applied in multilayered 3D erasable bit optical data storage.

Krul et al. [59] modified a photosensitive system based on polymethylmethacrylate (PMMA) by the additions of radical copolymerisation of methylmethacrylate (MMA) with acrylic acid (AA). This enhanced the adhesion to soda-lime glass substrate, thus also improving the heat and thermal stability of the resulting holographic gratings. Polymer films deposited from samples of the copolymer of MMA with AA containing 9,10-phenanthrenequinone additives were used as a photosensitive material for the recording of holographic gratings. Their results demonstrated that the system of copolymer of MMA with AA was more stable and only showed some deterioration at $200^{\circ} \mathrm{C}$. However, even in this case it was possible to measure the DE, which then fell to zero within 350 minutes. Therefore it was found possible to generate gratings that are thermally stable up to $200^{\circ} \mathrm{C}$ using this modified PMMA material.

The effective optical thickness of the material layer, $d_{\text {eff }}$, determines the width of the central lobe in the off-Bragg response of the gratings recorded, and this parameter therefore governs the angular separation needed between two consecutive holograms so that they may be read separately with low crosstalk during read out. Gallego et al. [60] proposed a new method to record many angularly multiplexed holograms, having similar diffraction efficiency values by taking into account the different effective optical thickness of each hologram. The method therefore predicts the exposure times required to obtain maximum storage capacity. AA/ PVA-based photopolymer material was studied in order to analyse the different dependence of the effective optical thickness on the number of holograms multiplexed.

Temperature changes during the recording process can have a profound influence on the hologram medium: (1) the exposing amount of energy to eliminate inhibiting by oxygen is altered; (2) the material sensitivity and $M / \#$ are varied; (3) the average refractive index changes and on-Bragg replay angle variations are found; (5) changes are founded in the monomer diffusion speed during the recording process. In 2008, Toishi et al. [61] proposed a compensation method to suppress the effects of temperature change. Through a description of how these parameters affect actual recording properties, methods to compensate for and thus avoid these problems were presented: (1) tuning of the preexposure energy; (2) using the proper recording schedule for a given temperature; (3) tuning the recording angle and wavelength; (5) recording each hologram at high speed. These methods then could be employed by actively detecting the medium temperature during recording. It was also found that the changes of the diffusion coefficient and the polymerization rate primarily affected parameters such as $M / \#$, the medium recording sensitivity, the preexposure time, and the transmission rate.

Khan et al. [62] reported an efficient and solventless processing technique for the fabrication of millimeter-thick, high-quality optical films for holographic storage based on a nondiffusion crosslinked photopolymer material. This was achieved by incorporation of crosslinkable Dewar benzene derivatives, which allowed the production of thick films 
that exhibit no diffuse light scattering and low absorption cross-section at the desired recording wavelength. A variety of photochemical reactions can be utilized to achieve such refractive index modulation. However, very few reactions exhibit quantum yields greater than unity, that is, where one photon of light triggers numerous chemical reactions. This nonlinearity and efficiency are essential for the high sensitivity and ultrafast recording speeds required for commercial holographic data storage. In examining other photochemical reactions with quantum yields greater than unity, the isomerization of Dewar benzene was noteworthy due to its very high quantum yield ( $>100$ in solution). Additionally, isomerization of Dewar benzene gave rise to a large change in the electronic structure of the molecule and was therefore anticipated to result in high refractive index modulation upon photoisomerization. Utilizing $1 \mathrm{~mm}$ thick films of the described recording medium, diffraction efficiencies of up to $100 \%$ at a recording intensity of $\sim 6 \mathrm{~mW} / \mathrm{cm}^{2}$ and $M / \#>6$ were obtained. This work suggested that Dewar benzene systems may be attractive candidates for writeonce-read-many (WORM) disk-based holographic memory devices.

In 2008, Matusevich et al. [63] further developed the material reported in [59] to investigate the contribution of the absorption and phase gratings to the total diffraction efficiency of volume holographic gratings, written in glasslike polymer recording materials, based on poly(methylmethacrylate) and a thermostable derivative (copolymer with acrylic acid) with distributed phenanthrenequinone (PQ-PMMA). Absorption by the recording media decreased following illumination and increased by heating. The maximal diffraction efficiency was 2\% (0.5\%) for absorption type gratings and $32 \%(22.5 \%)$ for phase gratings in PMMA+AA (PMMA). Refractive index modulations in the range $10^{-4}$ $10^{-3}$ were observed during recording. During heating the diffraction efficiency of the absorption grating could reach $4 \%$ for PMMA, while for absorption grating in PMMA+AA it was $0.3 \%$. The total diffraction efficiency in saturation was about $10 \%$ for both material samples.

McLeod et al. [64] demonstrated a three-dimensional optical data storage system in an initially homogenous volume by first recording a reflection grating in a holographic photopolymer. The volume data storage method used a single read/write head locked, in radius and depth, to a prefabricated volume servopattern. In addition, the power in the writing focus did not depend on depth, and the reflection efficiency of the bits was independent of the precise writing mechanism. Difficulties associated with the use of two opposing read/write heads were overcome by separating the holograms from the write mechanism so that a single-head optical drive could both read and write from one side of the disk. Superposition of two or three of such gratings, with slightly different $k$-vectors, created a track and layer structure that specialized servodetection optics can use to lock the focus to these deeply buried tracks. Writing was accomplished by locally modifying the reflectivity of the preexisting hologram. This modification could take the form of ablation, inelastic deformation via heating at the focus, or erasure via linear or two-photon continued polymerization in the previously unexposed fringes of the hologram. Moreover, by fabricating the precision grating in the factory, the drive does not require vibration isolation or a highly coherent laser, both hallmarks of traditional holographic data storage. This work suggested the strong possibility of TByte capacity in submillimeter thickness disks of diameter $120 \mathrm{~mm}$.

Gallego et al. [65] reported on the different values of shrinkages or swelling of photopolymer layer by using a reflection interferometer. Furthermore, these variations were shown to depend on the spatial frequencies of the gratings stored in the materials. Using this technique they measured the variations of the layer refractive index and estimated the polymer refractive index. Their results suggested that at higher spatial frequencies, monomer diffusion from the dark zones to the bright ones, together with surface tension effects, could considerably affect the material volume changes. In particular, molecular migration (mass transport) reduced shrinkage. Values of shrinkage of around 2\% (without crosslinker) and 3\% (with crosslinker) were obtained. This reduction was observed to increase with the spatial frequency and with the monomer diffusion velocity.

Tomita et al. [66] examined thermal distortions of volume holograms recorded in (meth)acrylate photopolymers doped with $\mathrm{SiO}_{2}$ or $\mathrm{ZrO}_{2}$ nanoparticles. A holographic method was used to evaluate the temperature-induced Bragg-angle detuning of the recorded volume holograms as a result of thermally induced refractive index and dimensional changes. It was shown that thermal distortions could be suppressed by reducing both the thermooptic coefficients $d n / d T$ and the out-of-plane linear coefficient of thermal expansion, by the addition of inorganic nanoparticles to the photopolymer. A reduction in the out-of-plane thermal expansion by approximately a factor of 2 was observed. Such an improvement, besides both extending the range of operating temperatures (the increase in $\Delta n$ ) and suppressing polymerization-related shrinkage, suggested the value of nanoparticle-polymer composite materials not only for holographic data storage but also for other photonic applications, that is, for diffractive optical element fabrication, in optical interconnects, for narrowband optical filters, and for using displays.

One of the key predictions of the NPDD model $[49,50]$ is that a reduction in the extent of the nonlocal effects within a material will improve its high spatial frequency response. In the work presented in 2008 by Gleeson et al. [67], the spatial frequency response of an acrylamide/polyvinyl alcohol-based photopolymer was improved through the addition of a chain transfer agent (CTA), sodium formate. The CTA has the effect of decreasing the average length of the polyacrylamide (PA) chains formed, thus reducing the nonlocal response parameter, $\sigma$ while maintaining a high rate of polymerisation. In addition, this work indicated that the chain transfer kinetic effects introduced by the CTA, which contribute to the increased localization of the polymerization, include (a) an increase in the concentration of the monomer radicals available for bimolecular termination and/or (b) the effects of less than $100 \%$ efficiency in the reinitiation process. A significant reduction in the nonlocal parameter, from 63 to $50 \mathrm{~nm}$, was obtained. 
Kelly et al. [68] proposed an algorithm, using a more rigorous NPDD formulation, to determine an appropriate holographic data storage recording schedule based on the physical properties of the recording medium's properties. The predictions of the algorithm and the inverse-square scaling law of holographic diffraction were examined experimentally, for a peristrophic multiplexing scheme. The scaling law was shown to significantly break down for low numbers of high diffraction efficiency gratings. Using the algorithm, a good correlation between the theoretically predicted and experimentally achieved exposure schedules was found. The largest discrepancy in all cases occurs for the final exposure, where the reduced monomer concentration available, and possible grating nonuniformities, strongly affects the exposure schedule time. The examinations showed that for multiple high diffraction efficiency gratings the scaling relationship, between the numbers of gratings recorded, broke down but that this could be explained using Kogelnik's model.

In 2009, Choi et al. [69] proposed a new free radical polymerization holographic recording medium, based on low shrink cyclic allylic sulfides (LS-CASs) ring-opening monomers. For comparison, the LS-CAS monomer was replaced with 4-bromostyrene. In ring-opening systems, a covalent bond is broken within the molecule for every covalent bond formed between the molecules. The result is the paradoxical situation of there being no net bond formation during the polymerization, and therefore ring-opening monomers typically display at least half the shrinkage of a conventional monomer of the same molecular weight. In this material system, 6-methyl-3-methylene-1,5-dithiacyclooctane was used as monomer. The percentage of volume shrinkage is measured to be $0.02 \%$, with refractive index contrast of (1.01 \pm $0.5) \times 10^{-3}$. This result indicated that a fivefold decrease in the volume shrinkage compared to 4-bromostyrene monomer system.

Orlic et al. [70] examined different classes of organic photosensitive materials in order to optimise the interaction between the material and the surrounding read/write optoelectronic system. Both cationic ring-opening and free radical polymerization systems were investigated. Localised recording of small volume reflection holographic elements, that is, microgratings, enabled testing of a variety of exposure conditions with a single record, allowing rapid optimisation of the exposure conditions. For the purpose of testing and investigating different photosensitive polymer materials, a laboratory system was developed that allows for high flexibility in their optical characterization experiments. While the microlocalised recording setup was designed for recording/readout at the optical resolution limit, the media tester comprises objective lenses having lower numerical apertures, and it was designed to operate with microgratings larger than a few hundred nanometers. Methods of investigation include spatial, spectral, and temporal measurements of the reflection diffraction efficiency at the individual recorded microgratings. In this way, an optimised exposure scheduling was achieved. The results confirmed that careful investigation and optimisation of the exposure scheduling is necessary in order to best use a specific photopolymer material.
Lin et al. [71] fabricated two photopolymer samples, Irgacure 784 doped poly(methyl methacrylate) (PMMA) and Irgacure 784 doped epoxy resin, using a two-step thermopolymerization procedure. Comparing the experimental results for Irgacure 784/PMMA to those produced using PQ/PMMA, the $M \#$ was improved by a factor of 1.9 , and the sensitivity has been improved by a factor of 10 . These experimental results revealed that Irgacure 784 molecules are efficient in increasing the holographic recording sensitivity at $532 \mathrm{~nm}$. By adding acrylamide (AA) and 2-hydroxyethyl methacrylate (HEMA) monomers, together with photosensitive DI and Irgacure 784 to form the doping solution, and then using diglycidyl ether (BADGE) and $N$-(2-Aminoethyl)-3-aminopropyl-trimethoxysilane (3ABTS) together to form a polymer matrix solution, they fabricated Irgacure 784 doped epoxy resin photopolymer. It was found that the $M \#$ and sensitivity of the Irgacure 784 doped epoxy resin are 5.49 and $26.65 \mathrm{~cm}^{2} \mathrm{~J}^{-1}$, respectively. The $M \#$ was around 1.7 times larger, whereas the sensitivity was about 350 times higher than that of Irgacure 784/PMMA sample. These results suggested that epoxy-resin matrix-based photopolymer system provides an effective way to improve the holographic recording characteristics for volume data storage.

To improve the temperature tolerance of holographic data storage systems, Tanaka [72] proposed a compensation method adjusting the wavelengths and incident directions of the recording and reading beams. The reading temperature tolerance of a photopolymer medium recorded at $25^{\circ} \mathrm{C}$ and the tolerance of the recording and reading temperature are calculated using the compensation method. Typically, the reading tolerance is from -20.0 to $+26.0 \mathrm{~K}$, and the recording and reading tolerance is $\pm 10.0 \mathrm{~K}$. This suggested that, to widen the tolerance, it was effective to increase the coefficient of thermal expansion (CTE) of the substrate or to decrease the CTE of the photopolymer, thereby reducing the difference of the CTEs.

In 2009, Kim and Chung [73] optimised a photopolymer system made from complex compositions of binder, photoacid generator (PAG), and sensitiser involving the cationic ring-opening polymerization of 1,3-bis[2-(3-7oxabicyclo-[4.1.0]heptyl)]-tetramethyldisiloxane in the presence of polydimethylsiloxane, with four epoxide moieties as a comonomer. It was found that when diffractive efficiency values were compared quantitatively, in order to analyse the effect of the binder on holographic photopolymerisation, the diffractive efficiency was affected by the viscosity of the mixture. Extremely low diffractive efficiency values were observed when the immiscible dimethyl silicone was used as a binder. The optimal conditions were also given in this work as a binder viscosity between 250 to $390 \mathrm{cP}$, and binder, PAG, and sensitiser content of $75-125 \mathrm{wt} \%,>6 \mathrm{wt} \%$, and $0.05 \mathrm{wt} \%$ compared to the total monomer mixture, respectively. This work suggested that a chemical, which is miscible with the monomer mixture, should be chosen as the binder when carrying out further studies to improve this photopolymer system.

All multilayer optical data storage methods, in which bits are written in an initially homogeneous material, are constrained by the imaging system phase aberrations that 
decrease the Strehl ratio (SR) as the number of layers and the index perturbation (due to each bit stored) are increased. Due to the complexities of accurately modelling the signal in such systems (e.g., due to consumption of the materials dynamic range out of focus exposures) and noise (e.g., due to wavefront variance), many studies of $3 \mathrm{D}$ data storage in homogeneous media were carried out experimentally. Without suitable relationships indicating how a particular experimental result can be extrapolated, comparison of different technological approaches is difficult. In 2009, McLeod [74] carried out an investigation to establish the capacity limitation on storage density with sufficient generality so that it can be applied independently of the particular system: (i) write mechanism, (ii) modulation code, (iii) nonlinearity of the material response, (iv) details of the lens, and (v) the disk geometry. The resulting equations for the Strehl ratio (SR) of a spot focused through the disk are quite simple and independent of the specific storage mechanism. Importantly, they revealed several interesting scaling relationships. Such scalings, even though derived via a number of approximations, provide a valuable method to evaluate and optimise next-generation optical storage methods. It is noted that the observed noise on the microholographic signal does not appear to be due to changes in the induced wavefront aberration and thus must arise due to other qualities of the material or optical system.

Gallego et al. [75] examined the influence of diffusion in polyvinyl-alcohol/acrylamide at very low spatial frequencies, that is, for spatial frequencies between 2 and 6 lines $/ \mathrm{mm}$, and also the dark evolution of the grating shape. They demonstrated the influence of the spatial frequency on the magnitude and sign of the material volume variations. In this work, they indicated that if the diffusion phenomena were well predicted, it was possible to achieve diffractive gratings with very high values of diffraction efficiency in the first order (near 35\%). These results are a significant preliminary step to build diffractive elements with stable properties using PVA/AA-based photopolymers.

Liu et al [76] reported on the fabrication of centimetersize transmission Bragg grating structure in semiconductor CdSe quantum dots dispersed in $50 \mu \mathrm{m}$ thick photopolymer films. This was done by holographic assembly of CdSe quantum dots in a photopolymerisable monomer blend. This single-step holographic method, involving the so-called holographic assembly of nanoparticles in polymer, does not require a shadow mask and can fabricate centimetersize multidimensional photonic lattice structures. Periodic patterning of CdSe quantum dots in polymer was confirmed by a fluorescence microscope and confocal Raman imaging. Because of the high refractive index of CdSe QDs and their high thermal mobility, the diffraction efficiency from the grating of period $1 \mu \mathrm{m}$ was close to $100 \%$ in the green with 0.34 vol\% CdSe quantum dots, giving a refractive index modulation as large as $5.1 \times 10^{-3}$ at a recording intensity of $100 \mathrm{~mW} / \mathrm{cm}^{2}$.

In 2010, Gleeson et al. [16] further developed the nonlocal photopolymerization-driven diffusion (NPDD) model to more accurately model the effects of (i) time-varying primary radical production, (ii) the rate of removal of photosensitizer, and (iii) inhibition. For the first time, the spatial and temporal variations in primary radical generation were included. These extensions provide a more physically comprehensive theoretical representation of the processes, which occur during free radical photopolymerization. The model was also extended to incorporate the effect of oxygen diffusion from outside the material layer by including a rate of oxygen replenishment from the surrounding environment. This allowed accurate modelling of the inhibition effects, which dominate the start of grating growth. This work provided a comprehensive approach to analyse and optimise the photopolymer materials under low intensity condition.

Omura et al. [77] further developed their nanoparticlepolymer composite material by optimising the concentration of $\mathrm{ZrO}_{2}$ nanoparticles at a wavelength of $404 \mathrm{~nm}$. The dependence of the polymerization kinetics on the concentrations of initiator and $\mathrm{ZrO}_{2}$ nanoparticles in acrylate monomer was examined using a photodifferential scanning calorimeter. The optimum concentration of $\mathrm{ZrO}_{2}$ nanoparticles to maximise the polymerization rate was identified. The diffraction and scattering properties of transmission volume gratings were also investigated. Refractive index modulations and the material recording sensitivities as high as $8 \times 10^{-3}$ and $9000 \mathrm{~cm}^{2} / \mathrm{J}$ were demonstrated at the optimum $\mathrm{ZrO}_{2}$ nanoparticle concentration of 35 vol. $\%$ and at a recording intensity of $5 \mathrm{~mW} / \mathrm{cm}^{2}$. The occurrence of the maximum photopolymerisation rate later during conversion accelerates the mutual diffusion of monomer molecules and nanoparticles during recording. This results in an increase in $\Delta n_{\text {sat }}$. It was also observed that the sensitivity dramatically increases at the shorter wavelengths, for example, $404 \mathrm{~nm}$, being approximately seven times higher than that at $532 \mathrm{~nm}$ at a recording intensity of $10 \mathrm{~mW} / \mathrm{cm}^{2}$.

Later in that year, Hata and Tomita [78] proposed a novel nanoparticle-polymer composite to further suppress shrinkage of their material using of the thiol-ene step-growth polymerization mechanism. Due to crosslinked thiol-ene, polymerisation proceeds very rapidly but will not reach the gel point until high functional group conversions take place, that is, reaching the gelation point later during conversion in the mixtures accelerates the mutual diffusion and phase separation of the monomer molecules and nanoparticles during recording. This will again result in an increase in $\Delta n_{\text {sat }}$ and the material sensitivity. It was shown that the polymerisation shrinkage can be reduced as low as $0.3 \%$ for a nanoparticle concentration of $35 \mathrm{vol} . \%$, giving a maximum refractive index modulation and material sensitivity of $8 \times 10^{-3}$ and $1014 \mathrm{~cm} / \mathrm{J}$, respectively, when recording in the green. These results provide a significant and noticeable advance in the development of holographic data storage materials.

Guo et al. [79] examined the photosensitiser diffusion effects of erythrosine $\mathrm{B}$ (EB) in an acrylamide/polyvinyl alcohol (AA/PVA) material. This was achieved using simple experimental techniques and use of a $2 \mathrm{D}$ diffusion model. The rate of $\mathrm{EB}$ diffusion, $\mathrm{D} \approx 6.27 \times 10^{-12} \mathrm{~cm}^{2} / \mathrm{s}$, was estimated. In the literature [80-82], the rate of diffusion of the acrylamide monomer (AA) in such photopolymer layers has been reported to be in the range $\sim 10^{-11}-10^{-10} \mathrm{~cm}^{2} / \mathrm{s}$. 
The molecular weight of EB is $879.86 \mathrm{~g} / \mathrm{mol}$ while that of AA is $71.08 \mathrm{~g} / \mathrm{mol}$. While molecular weight alone does not determine the rate of diffusion, it would be reasonable to expect EB to diffuse more slowly than AA. The results indicated that $\mathrm{EB}$ diffuses an order of magnitude slower than the AA monomer.

Sabol et al. [83] then reported a detailed study on the photoinitiation process of the photosensitizer, Irgacure 784, used in the epoxy-resin-based photopolymer. Irgacure 784 is a type of the titanocene photoinitiator which has the property that it does not require a coinitiator to produce the free radical. In this work, the photochemical reactions of Irgacure 784 involved are thoroughly discussed. Importantly, the experimental results reported verify that multiple different absorbers are simultaneously present in the layer at different stages during the exposure.

In 2011, Hata et al. [84] introduced the thiol-ene monomers into silica nanoparticle-polymer composites and examined the photopolymerisation kinetics and volume holographic recording characteristics. Real-time Fourier transform spectroscopy and photocalorimetry were used to characterize the visible light curing kinetics containing a secondary dithiol (with high self-life stability and low odor) and a triene with rigid structure and high electron density. It was shown that while the nanoparticle-(thiol-ene) polymer composites exhibit high transparency, values of $\Delta n_{\text {sat }}$ and $S$ as large as $1 \times 10^{-2}$ and $1615 \mathrm{~cm}^{2} / J$ were achieved. The polymerisation shrinkage was reduced to the value as low as $0.4 \%$, as a result of delaying the gelation point in conversion by introducing the thiol-ene monomers. These values were within the acceptable range of values for holographic data storage media (i.e., $\Delta n_{\text {sat }}=5 \times 10^{-3}, S=500 \mathrm{~cm}^{2} / \mathrm{J}$ and $0.5 \%$ shrinkage). It was also shown that because of the dispersion of the inorganic silica nanoparticles and the use of the triene monomer, (having the rigid structure of the triazine functional group), the thermal stability of the recorded holograms is much improved compared to that of those previously reported in earlier nanoparticle-polymer composites using organic nanoparticles and primary mercaptopropionate trithiol/allyl ether triene monomers [53, 78].

Further research to the properties of the stoichiometric thiol-to-ene and nanoparticle-(thiol-ene)polymer composites was presented by Hata and Tomita [85] later in the same year. The impact of the stoichiometric thiol-ene ratio on the properties of photopolymerisation was estimated. It was found that the thiol-ene stoichiometry strongly influences the dynamics of the thiol and ene functional group conversions and therefore the polymerisation rate, $R_{p}$, for samples with silica nanoparticle dispersion. It was also found that $\alpha_{c}$, which is the value of time-dependent relative conversion $\alpha$ at the peak value of $R_{p}$, increases with increasing stoichiometric ratios of thiol-to-ene functional groups, $r$. This work indicated that while $\Delta n_{\text {sat }}$ and $S$ are maximized at $r=1$, out-of-plane fractional thickness change, $\sigma$, can be reduced by increasing $r$. It was also shown that there exists a negative correlation between $\alpha_{c}$ and $\sigma$. It suggested that such a correlation must also be presented in other holographic photopolymer systems, and that can be used as part of the material design strategy for holographic applications.
Certain photorefractive processes, as is the case for photopolymerisation, involve mass transport. As a consequence, not only the optical but also the neutron refractive index may be changed in these cases [86]. The light-induced change of the refractive index for neutrons in materials (the photoneutron-refractive effect) has been exploited to produce diffraction gratings for neutrons by structuring suitable recording materials using holography [87]. The reduction in size of the grating modulation with position, that is, within the layer depth due to the absorption during the recording process, becomes an issue at grating thicknesses of about 100 microns and larger. This limits the achievable diffraction efficiency for neutrons. In an attempt to overcome this problem, the Pendellosung interference effect in holographic gratings was examined by Klepp et al. [88] in 2011. Diffraction experiments with holographic gratings recorded in $\mathrm{SiO}_{2}$ nanoparticle-polymer composites were carried out with slow neutrons. The influence of parameters, such as the nanoparticle concentration, grating thickness, and grating spacing, on the neutron-optical properties of such materials was tested. It was shown that the difficulty of obtaining sufficiently larger thicknesses can be overcome by exploiting the Pendellosung interference effect. This was done by tilting the grating around an axis parallel to the grating vector to increase the effective thickness of the gratings. Using this method, high peak reflectivity of $83 \%$ was achieved and reached for a neutron wavelength of $3.76 \mathrm{~nm}$. Such results, besides extending the range of passive neutron optical elements available, have also provided evidence for the superiority of the Betavalue method over Kogelnik's $K$-vector closure method [89, 90].

In work by Vayalamkuzhi et al. [91], a panchromatic silver-doped poly (vinyl alcohol)/acrylamide photopolymer system was developed. In the presence of $\mathrm{Ag}^{+}$, diffraction efficiency of $70 \%$ in transmission gratings was recorded using both $\mathrm{Ar}^{+}$and Nd:YAG lasers. This offers the possibility of using the developed photopolymer system as a panchromatic recording medium. Such material developments have important applications in the fields of true-color display holography, holographic storage, and holographic optical elements.

The microholographic approach relies on submicronsized reflection gratings recorded in layer at different depths to encode the digital data stored. In 2011, Orlic et al. [92] presented an optical write/read system for high density optical data storage in 3D. This design of their optical system for high-density microholographic storage was based on a single-beam path, using a retroreflecting configuration. The combined performance of these two components, that is, a retroreflector and confocal filter, was a key feature of the system. They demonstrated resolution-limited volume recording in photopolymer materials sensitive in the green and violet spectral ranges. The optimum exposure condition was identified as a compromise between the requirements of strong volume localization and sufficiently high diffraction efficiency. Microgratings with widths of $306 \mathrm{~nm}$ at $532 \mathrm{~nm}$ and $197 \mathrm{~nm}$ at $405 \mathrm{~nm}$ were recorded. These results were the smallest volume microholograms ever recorded up to that time. Photopolymer materials used in this work 
were cationic ring-opening polymerization (CROP) systems developed by Aprilis [93]. The work indicated the potential of the technique for volumetric optical structuring, data storage, and encryption applications.

Following on from the work by Ortuño et al. [94] in 2007, on the development of a nontoxic photopolymer, Gallego et al. [95] further developed a new environmental sustainable photopolymer, by introducing a sodium acrylate (NaAO) into AA/PVA-based photopolymer material as a substitute for acrylamide-based photopolymers in the manufacture of diffractive optical elements. Diffraction efficiencies of $\sim 40 \%$ were achieved for planar gratings. They estimated the $\mathrm{NaAO}$ diffusion rate inside the photopolymer, and the values obtained were 10 times smaller than those in the case of the widely studied PVA/AA photopolymer. Therefore the PVA/NaAO photopolymer should be used when slow diffusion is required. The influence of TEA on monomer diffusion inside the material and on the polymerisation rate was also examined. The results suggested that the concentration of this component, that is, $\mathrm{NaAO}$, can be carefully adjusted in order to obtain photopolymers with desired dynamic properties.

Following this work [95], later in the same year, Gallego et al. [96] reported on other possibilities to achieve thin photopolymer layers with lower environmental impact. Using direct measurements of the phase shift and the shrinkage of photopolymeric layers based on $\mathrm{PVA} / \mathrm{NaAO}$ material (for five different chemical compositions), they estimated several different material parameters. They obtained values of the shrinkage between $1 \%$ and $3 \%$ in the layers analysed. Although the results for shrinkage were too high for holographic memory applications, these values are promising for use in obtaining relief structures for liquid crystal applications. From comparison of the results to those for typical PVA/AA-based materials, it was shown that sodium acrylate provides less toxic photopolymer thin layers suitable for very low spatial frequency applications. It was also suggested that, in order to achieve "greener" layers [94], the incorporation of sodium salt $5^{\prime}$-riboflavin monophosphate (PRF) as a dye should be pursued.

Clearly, when attempting to improve a photopolymer material's performance for a given application, an understanding of the photochemical and photophysical processes, which occur during photopolymerisation, is extremely important. In 2011, Gleeson et al. [97] demonstrated how NPDD-based modelling of the mechanisms which occur in photopolymers during and after exposure has led to the development of a tool, which can be used to predict the behaviour of many distinct types of material for a wide range of recording conditions. They reported on work to the extended their NPDD model, in order to clearly quantify some of the trends, which the model predicts, and thus be able to analyse their implications for attempts to improve photopolymer material performance. The results are of practical importance when attempting to optimise the performance of a photopolymer material. As the many types of monomer have diverse chemical and structural characteristics, knowledge of these characteristics is necessary. For example, when choosing a monomer, an informed choice can yield specific improvements in material performance. One of the implications of the predictions of the NPDD model is that utilising a monomer with a large propagation rate constant and low bimolecular termination rate will produce a higher refractive index modulation, $\Delta n_{\text {sat }}$. However, it is also desirable to have a monomer with high mobility, that is, a fast diffusion rate, in order to increase the dynamic range of the photopolymer and to maximise the index modulation achievable. One of the results highlighted in this work is that if the propagation rate is too large or the bimolecular termination rate is too small, the optimum refractive index modulation will not be obtained. These deleterious effects are compounded further by increased material viscosity as a result of polymerisation. In this work a qualitative understanding of trends is given for quantitive predictability.

Gleeson et al. [98] reported results for an impressive new acrylate-based photopolymer material developed by Bayer Material Science (BMS). The material was examined using various optical techniques and then characterised using the NPDD model. Refractive index modulation up to $\Delta n_{\text {sat }}=8 \times$ $10^{-3}$ and a very low nonlocal parameter value of $9.2 \mathrm{~nm}$ were estimated. A comparison between AA/PVA-based and BMS materials shows that the BMS material has (i) a substantially faster response of the refractive index modulation with respect to the recording dosage, especially at lower power densities; (ii) three times higher refractive index modulation achievable; (iii) six times smaller nonlocal response parameter, (representing the spatial spread of the reactive chain ends of the formed polymer coils during photopolymerisation), indicating a much higher resolution, that is, little dropoff in material response up to 5000 lines/mm; (iv) an improved performance at high spatial frequencies permitting high diffraction efficiency reflection holograms to be recorded. This material demonstrated the capabilities of a new class of photopolymer, which importantly can be produced on an industrial scale as large area plastic films, offering high index modulation, full colour recording, high light sensitivity, and environmental stability.

The NPDD model has been extended to include the kinetics of chain transfer and reinitiation, in order to analyse the effects of various chain transfer agents on the system kinetics and to study their use in reducing the average polymer chain length [67], in free-radical-based photopolymer materials. Guo et al. $[99,100]$ studied AA/PVA-based photopolymer material containing chain transfer agents. The effects of two different types of chain transfer agent, that is, Sodium Formate (HCOONa) and 1-Mercapto-2-Propanol $\left(\mathrm{CH}_{3} \mathrm{CH}(\mathrm{OH}) \mathrm{CH}_{2} \mathrm{SH}\right)$, were compared. The validity of the extended NPDD model $[99,100]$ was examined by applying it to fit the resulting experimental data. It is confirmed that the average polymer chain length formed is reduced by the addition of the chain transfer agents, as they reduce the nonlocal response of the materials. The most effective material combination used resulted in a reduction in the nonlocal parameter, from $61 \mathrm{~nm}$ to $41 \mathrm{~nm}$. Comparing the the two types of transfer agents, the results achieved using 1mercapto-2-propanol were shown to be better than those using sodium formate. These CTAs produced improved high spatial frequency material response, giving up to $\sim 28 \%$ 
increase in the refractive index modulation achieved at 3000 line $/ \mathrm{mm}$.

\section{Conclusions}

An overview of reported work on the optimisation of photopolymer materials is presented, and several types of photopolymers are discussed. We have emphasised in this review those materials used as holographic data storage (HDS) media. Even limiting our review to papers explicitly dealing with such photopolymer materials, it is clear that there have been impressive developments in the field of photopolymer material research since photopolymers were first studied as a holographic recording media in 1969 [27]. As described various types of photopolymer for use with a range of read/write technologies have been developed. Increased material sensitivities, resolutions, diffraction efficiencies, and material stabilities have been reported. Such improvements include (i) two-photon absorption techniques to store and retrieve information in the volume of the material layer [58]; (ii) the introduction of nanoparticles to reduce polymerisation shrinkage [35]; (iii) the introduction of thermally curable matrix networks suitable for mass production $[32,98]$; (iv) the introduction of chain transfer agents to improve the high spatial frequency performance of the materials $[67,99,100]$; (v) the introduction of quantum dots to improve the materials' refractive index modulation and fluorescence $[58,76]$; (vi) the introduction of PMMAbased photopolymer materials to increase the thermal stability [59]. Clearly many promising materials, providing high storage capacities, are being developed for the next generation optical data storage media.

Photopolymer materials offer many advantages; however, there still exists several detrimental effects encountered in those materials as they are being optimised for commercial applications. What this paper clearly leads us to conclude is that it is important to build up a physically comprehensive model, which can be used to predict the behaviours of these materials, in order to improve their performance and reduce the effects that limit their commercial use. In order to achieve this comprehensive model, a number of effects must be included; these are (a) multicomponent diffusion; (b) viscosity effects; (c) shrinkage; (d) inhibition; (e) mass transport effects; (f) spatially nonlocal polymer chain growth; (g) spatial/temporal primary radical (photokinetic) behaviours; (h) thermal effects. As this model develops, it will provide a more rigorous and informed basis for predicting the behaviours of photopolymer materials and will indicate useful trends in performance, which can be used for material optimisation $[97,101]$.

\section{Acknowledgments}

One of the authors (J. Guo) currently holds an IRCSET Government of Ireland Postgraduate Scholarship in Science, Engineering and Technology, while another author (M. Gleeson) currently holds a Government of Ireland Postdoctoral Scholarship in Science, Engineering and Technology, also granted by the Irish Research Council for Science, Engineering and Technology. The authors would also like to acknowledge the support of Enterprise Ireland and Science Foundation Ireland under the National Development Fund.

\section{References}

[1] http://www.insic.org/.

[2] D. Sarid and B. H. Schechtman, "A roadmap for optical data storage application," Optics and Photonics News, vol. 18, no. 5, pp. 32-37, 2007.

[3] Aprilis 2007, http://www.hedgewood.com/.

[4] InPhase Technologies 2007, http://www.inphase-technologies.com/, Tapestry Media.

[5] K. Curtis, L. Dhar, L. Murphy, and A. Hill, "Future developments," in Holographic Data Storage: From Theory to Practical Systems, Wiley, New York, NY, USA, 2010.

[6] H. J. Coufal, D. Psaltis, and G. T. Sincerbox, Holographic Data Storage, Springer, New York, NY, USA, 2000.

[7] C. Ye and R. R. McLeod, "GRIN lens and lens array fabrication with diffusion-driven photopolymer," Optics Letters, vol. 33, no. 22, pp. 2575-2577, 2008.

[8] A. Sato, M. Scepanovic, and R. K. Kostuk, "Holographic edge-illuminated polymer Bragg gratings for dense wavelength division optical filters at $1550 \mathrm{~nm}$," Applied Optics, vol. 42, no. 5, pp. 778-784, 2003.

[9] A. B. Villafranca and K. Saravanamuttu, "Diffraction rings due to spatial self-phase modulation in a photopolymerizable medium," Journal of Optics A, vol. 11, no. 12, Article ID $125202,2009$.

[10] G. Manivannan and R. A. Lessard, "Trends in holographic recording materials," Trends in Polymer Science, vol. 2, pp. 282-290, 1994.

[11] J. R. Lawrence, F. T. O’Neill, and J. T. Sheridan, "Photopolymer holographic recording material," Optik, vol. 112, no. 10, pp. 449-463, 2001.

[12] F. K. Bruder and F. Thomas, "Materials in optical data storage," International Journal of Materials Research, vol. 101, no. 2, pp. 199-215, 2010.

[13] G. Odian, Principles of Polymerization, Wiley, New York, NY, USA, 4th edition, 1991.

[14] M. R. Gleeson and J. T. Sheridan, "Nonlocal photopolymerization kinetics including multiple termination mechanisms and dark reactions. Part I. Modeling," Journal of the Optical Society of America B, vol. 26, no. 9, pp. 1736-1745, 2009.

[15] M. R. Gleeson, S. Liu, R. R. McLeod, and J. T. Sheridan, "Nonlocal photopolymerization kinetics including multiple termination mechanisms and dark reactions. Part II. Experimental validation," Journal of the Optical Society of America $B$, vol. 26, no. 9, pp. 1746-1754, 2009.

[16] M. R. Gleeson, S. Liu, J. Guo, and J. T. Sheridan, "Non-local photo-polymerization kinetics including multiple termination mechanisms and dark reactions: part III. Primary radical generation and inhibition," Journal of the Optical Society of America B, vol. 27, no. 9, pp. 1804-1812, 2010.

[17] S. Liu, M. R. Gleeson, J. Guo, and J. T. Sheridan, "Optical characterization of photopolymers materials: theoretical and experimental examination of primary radical generation," Applied Physics B, pp. 1-11, 2010.

[18] A. Fimia, N. Lopez, F. Mateos, R. Sastre, J. Pineda, and F. Amat-Guerri, "Elimination of oxygen inhibition in photopolymer systems used as holographic recording materials," Journal of Modern Optics, vol. 40, no. 4, pp. 699-706, 1993. 
[19] F. R. Mayo, "Chain transfer in the polymerization of styrene: the reaction of solvents with free radicals," Journal of the American Chemical Society, pp. 2324-2329, 1943.

[20] M. Fevola, R. Hester, and C. McCormack, "Molecular weight control of polyacyrlamide with sodium formate as a chaintransfer agent: characterization via size exclusion chromatography/multi-angle laser light ccattering and determination of chain-transfer constant," Journal of Polymer Science A, vol. 41, no. 4, pp. 560-568, 2003.

[21] F. Karasu, N. Arsu, and Y. Yagci, "2-mercapto thioxanthone as a chain transfer agent in free-radical polymerization: a versatile route to incorporate thioxanthone moieties into polymer chain-ends," Journal of Applied Polymer Science, vol. 103, no. 6, pp. 3766-3770, 2007.

[22] H. Kogelnik, "Coupled wave theory for thick holographic gratings," Bell System Technical Journal, vol. 48, no. 9, pp. 2909-2947, 1969.

[23] M. G. Moharam and T. K. Gaylord, "Rigorous coupled-wave analysis of planar-grating diffraction," Journal of the Optical Society of America, vol. 71, no. 7, pp. 811-818, 1981.

[24] R. R. A. Syms, Practical Volume Holography, Clarendon, Oxford, Miss, USA, 1990.

[25] M. R. Gleeson and J. T. Sheridan, "A review of the modelling of free-radical photopolymerization in the formation of holographic gratings," Journal of Optics A, vol. 11, no. 2, Article ID 024008, 2009.

[26] F. T. O’Neill, J. R. Lawrence, and J. T. Sheridan, “Automated recording and testing of holographic optical element arrays," Optik, vol. 111, no. 10, pp. 459-467, 2000.

[27] D. H. Close, A. D. Jacobson, J. D. Margerum, R. G. Brault, and F. J. McClung, "Hologram recording on photopolymer materials," Applied Physics Letters, vol. 14, no. 5, pp. 159-160, 1969.

[28] N. Sadlej and B. Smolinska, "Stable photo-sensitive polymer layers for holography," Optics and Laser Technology, vol. 7, no. 4, pp. 175-179, 1975.

[29] S. Calixto, "Dry polymer for holographic recording," Applied Optics, vol. 26, pp. 3904-3909, 1987.

[30] S. Blaya, R. Mallavia, L. Carretero, A. Fimia, and R. F. Madrigal, "Highly sensitive photopolymerizable dry film for use in real time holography," Applied Physics Letters, vol. 73, no. 12, pp. 1628-1630, 1998.

[31] F. Zhao, E. E. E. Frietman, and X. Li, "Novel type of red sensitive photo-polymer system for optical storage," in Proceedings of the 1998 Conference on Advanced Optical Memories and Interfaces to Computer Storage, pp. 317-321, July 1998.

[32] T. J. Trentler, J. E. Boyd, and V. L. Colvin, "Epoxy resin photopolymer composites for volume holography," Chemistry of Materials, vol. 12, no. 5, pp. 1431-1438, 2000.

[33] M. Eich, J. H. Wendorff, B. Reck, and H. Ringsdorf, "Reversible digital and holographic optical storage in polymeric liquid crystals," Macromolecular Rapid Communications, vol. 8, no. 1, pp. 59-63, 1987.

[34] S. J. Zilker, M. R. Huber, T. Bieringer, and D. Haarer, "Holographic recording in amorphous side-chain polymers: a comparison of two different design philosophies," Applied Physics B, vol. 68, no. 5, pp. 893-897, 1999.

[35] N. Suzuki, Y. Tomita, and T. Kojima, "Holographic recording in $\mathrm{TiO}_{2}$ nanoparticle-dispersed methacrylate photopolymer films," Applied Physics Letters, vol. 81, no. 22, pp. 4121-4123, 2002.

[36] S. Blaya, L. Carretero, A. Fimia et al., "Optimal composition of an acrylamide and $\mathrm{N}, \mathrm{N}^{\prime}$-methylenebisacrylamide holographic recording material," Journal of Modern Optics, vol. 45 , no. 12 , pp. 2573-2584, 1998.
[37] S. Blaya, L. Carretero, R. Mallavia et al., "Optimization of an acrylamide-based dry film used for holographic recording," Applied Optics, vol. 37, no. 32, pp. 7604-7610, 1998.

[38] R. Mallavia, A. Fimia, S. Blaya et al., "A mixture of mono-, bi- and trifunctional acrylates with eosine O-benzoyl- $\alpha$ oxooxime: advances in holographic copolymerizable composition," Journal of Modern Optics, vol. 46, no. 4, pp. 559-566, 1999.

[39] A. Fimia, F. Mateos, R. Mallavia et al., "High-energy sensitivity enhancement in panchromatic photopolymers for holography using a mixture of visible-light photoinitiators," Journal of Modern Optics, vol. 46, no. 7, pp. 1091-1098, 1999.

[40] G. M. Karpov, V. V. Obukhovsky, T. N. Smirnova, and V. V. Lemeshko, "Spatial transfer of matter as a method of holographic recording in photoformers," Optics Communications, vol. 174, no. 5-6, pp. 391-404, 2000.

[41] F. T. O’Neill, J. R. Lawrence, and J. T. Sheridan, "Improvement of holographic recording material using aerosol sealant," Journal of Optics A, vol. 3, no. 1, pp. 20-25, 2001.

[42] H. Yao, M. Huang, Z. Chen, L. Hou, and F. Gan, "Optimization of two-monomer-based photopolymer used for holographic recording," Materials Letters, vol. 56, no. 1-2, pp. 3-8, 2002.

[43] Y. Tomita and H. Nishibiraki, "Improvement of holographic recording sensitivities in the green in $\mathrm{SiO}_{2}$ nanoparticledispersed methacrylate photopolymers doped with pyrromethene dyes," Applied Physics Letters, vol. 83, no. 3, pp. 410412, 2003.

[44] S. Blaya, P. Acebal, L. Carretero, and A. Fimia, "Pyrromethene-HEMA-based photopolymerizable holographic recording material," Optics Communications, vol. 228, no. 1-3, pp. 5561, 2003.

[45] M. Ortuno, S. Gallego, C. Garcia, C. Neipp, A. Belendez, and I. Pascual, "Optimization of a $1 \mathrm{~mm}$ thick PVA/acrylamide recording material to obtain holographic memories: method of preparation and holographic properties," Applied Physics $B$, vol. 76, no. 8, pp. 851-857, 2003.

[46] Y. H. Cho, M. He, B. K. Kim, and Y. Kawakami, "Improvement of holographic performance by novel photopolymer systems with siloxane-containing epoxides," Science and Technology of Advanced Materials, vol. 5, no. 3, pp. 319-323, 2004.

[47] R. R. McLeod, A. J. Daiber, M. E. McDonald et al., "Microholographic multilayer optical disk data storage," Applied Optics, vol. 44, no. 16, pp. 3197-3207, 2005.

[48] J. V. Kelly, M. R. Gleeson, C. E. Close et al., "Temporal analysis of grating formation in photopolymer using the nonlocal polymerization-driven diffusion model," Optics Express, vol. 13, no. 18, pp. 6990-7004, 2005.

[49] J. T. Sheridan and J. R. Lawrence, "Nonlocal-response diffusion model of holographic recording in photopolymer," Journal of the Optical Society of America A: Optics and Image Science, and Vision, vol. 17, no. 6, pp. 1108-1114, 2000.

[50] J. R. Lawrence, F. T. O’Neill, and J. T. Sheridan, "Adjusted intensity nonlocal diffusion model of photopolymer grating formation," Journal of the Optical Society of America B, vol. 19, no. 4, pp. 621-629, 2002.

[51] F. T. O’Neill, A. J. Carr, S. M. Daniels et al., "Refractive elements produced in photopolymer layers," Journal of Materials Science, vol. 40, no. 15, pp. 4129-4132, 2005.

[52] H. Wei, L. Cao, Z. Xu, Q. He, G. Jin, and C. Gu, "Orthogonal polarization dual-channel holographic memory in cationic ring-opening photopolymer," Optics Express, vol. 14, no. 12, pp. 5135-5142, 2006. 
[53] Y. Tomita, K. Furushima, K. Ochi et al., "Organic nanoparticle (hyperbranched polymer)-dispersed photopolymers for volume holographic storage," Applied Physics Letters, vol. 88, no. 7, 2006.

[54] B. M. John, M. Ushamani, R. Joseph, K. Sreekumar, and C. S. Kartha, "Reusable recording medium based on MBPVA and vinyl acetate," Journal of Modern Optics, vol. 53, no. 3, pp. 343-355, 2006.

[55] E. Fernandez, C. Garcia, I. Pascual, M. Ortuno, S. Gallego, and A. Belendez, "Optimization of a thick polyvinyl alcoholacrylamide photopolymer for data storage using a combination of angular and peristrophic holographic multiplexing," Applied Optics, vol. 45, no. 29, pp. 7661-7666, 2006.

[56] Y. C. Jeong, S. Lee, and J. K. Park, "Holographic diffraction gratings with enhanced sensitivity based on epoxy-resin photopolymers," Optics Express, vol. 15, no. 4, pp. 1497-1504, 2007.

[57] F. R. Ling, B. H. Tong, S. J. Jiang, B. Wang, and Y. L. Zhang, "Optimization of holographic storage with modulated recording beams in a thick polyvinyl alcohol/acrylamide photopolymer," Journal of the Optical Society of America A, vol. 24, no. 7, pp. 1945-1949, 2007.

[58] X. Li, C. Bullen, J. W. M. Chon, R. A. Evans, and M. Gu, "Two-photon-induced three-dimensional optical data storage in CdS quantum-dot doped photopolymer," Applied Physics Letters, vol. 90, no. 16, Article ID 161116, 2007.

[59] L. P. Krul, V. Matusevich, D. Hoff et al., "Modified polymethylmethacrylate as a base for thermostable optical recording media," Optics Express, vol. 15, no. 14, pp. 8543-8549, 2007.

[60] S. Gallego, M.F. Ortuno, C. Neipp, E. Fernandez, A. Belendez, and I. Pascual, "Improved maximum uniformity and capacity of multiple holograms recorded in absorbent photopolymers," Optics Express, vol. 15, no. 15, pp. 9308-9319, 2007.

[61] M. Toishi, T. Tanaka, and K. Watanabe, "Analysis of temperature change effects on hologram recordingand a compensation method," Optical Review, vol. 15, no. 1, pp. 11-18, 2008.

[62] A. Khan, G. D. Stucky, and C. J. Hawker, "High-performance, nondiffusive crosslinked polymers for holographic data storage," Advanced Materials, vol. 20, no. 20, pp. 3937-3941, 2008.

[63] V. Matusevich, A. Matusevich, R. Kowarschik, Y. I. Matusevich, and L. P. Krul, "Holographic volume absorption grating in glass-like polymer recording material," Optics Express, vol. 16, no. 3, pp. 1552-1558, 2008.

[64] R. R. McLeod, A. J. Daiber, T. Honda et al., "Threedimensional optical disk data storage via the localized alteration of a format hologram," Applied Optics, vol. 47, no. 14, pp. 2696-2707, 2008.

[65] S. Gallego, A. Marquez, D. Mendez et al., "Analysis of PVA/ AA based photopolymers at the zero spatial frequency limit using interferometric methods," Applied Optics, vol. 47, no. 14, pp. 2557-2563, 2008.

[66] Y. Tomita, T. Nakamura, and A. Tago, "Improved thermal stability of volume holograms recorded in nanoparticlepolymer composite films," Optics Letters, vol. 33, no. 15, pp. 1750-1752, 2008.

[67] M. R. Gleeson, D. Sabol, S. Liu, C. E. Close, J. V. Kelly, and J. T. Sheridan, "Improvement of the spatial frequency response of photopolymer materials by modifying polymer chain length," Journal of the Optical Society of America B, vol. 25, no. 3, pp. 396-406, 2008.
[68] J. V. Kelly, M. R. Gleeson, C. E. Close, and J. T. Sheridan, "Optimized scheduling for holographic data storage," Journal of Optics A, vol. 10, no. 11, Article ID 115203, 2008.

[69] K. Choi, J. W. M. Chon, M. Gu, N. Malic, and R. A. Evans, "Low-distortion holographic data storage media using freeradical ring-opening polymerization," Advanced Functional Materials, vol. 19, no. 22, pp. 3560-3566, 2009.

[70] S. Orlic, E. Dietz, T. Feid, S. Frohmann, and C. Mueller, "Optical investigation of photopolymer systems for microholographic storage," Journal of Optics A, vol. 11, no. 2, Article ID 024014, 2009.

[71] S. H. Lin, Y. N. Hsiao, and K. Y. Hsu, "Preparation and characterization of Irgacure 784 doped photopolymers for holographic data storage at $532 \mathrm{~nm}$," Journal of Optics A, vol. 11, no. 2, Article ID 024012, 2009.

[72] T. Tanaka, "Recording and reading temperature tolerance in holographic data storage, in relation to the anisotropic thermal expansion of a photopolymer medium," Optics Express, vol. 17, no. 16, pp. 14132-14142, 2009.

[73] D. Kim and D. W. Chung, "Study on the optimization of cationic ring opening polymerization of silicone-based epoxy monomers for holographic photopolymers," Macromolecular Research, vol. 17, no. 9, pp. 651-657, 2009.

[74] R. R. McLeod, "Impact of phase aberrations caused by multilayer optical data storage in weakly inhomogeneous media," Journal of the Optical Society of America B, vol. 26, no. 2, pp. 308-317, 2009.

[75] S. Gallego, A. Marquez, S. Marini, E. Fernandez, M. Ortuno, and I. Pascual, "In dark analysis of PVA/AA materials at very low spatial frequencies: phase modulation evolution and diffusion estimation," Optics Express, vol. 17, no. 20, pp. 18279-18291, 2009.

[76] X. Liu, Y. Tomita, J. Oshima et al., "Holographic assembly of semiconductor CdSe quantum dots in polymer for volume Bragg grating structures with diffraction efficiency near 100\%," Applied Physics Letters, vol. 95, no. 26, Article ID 261109, 2009.

[77] K. Omura and Y. Tomita, "Photopolymerization kinetics and volume holographic recording in $\mathrm{ZrO}_{2}$ nanoparticlepolymer composites at $404 \mathrm{~nm}$," Journal of Applied Physics, vol. 107, no. 2, Article ID 023107, 2010.

[78] E. Hata and Y. Tomita, "Order-of-magnitude polymerization-shrinkage suppression of volume gratings recorded in nanoparticle-polymer composites," Optics Letters, vol. 35, no. 3, pp. 396-398, 2010.

[79] J. Guo, S. Liu, M. R. Gleeson, and J. T. Sheridan, "Study of photosensitizer diffusion in a photopolymer material for holographic applications," Optical Engineering, vol. 50, no. 1, Article ID 015801, 2011.

[80] D. J. Lougnot, P. Jost, and L. Lavielle, "Polymers for holographic recording: VI. Some basic ideas for modelling the kinetics of the recording process," Pure and Applied Optics, vol. 6, no. 2, pp. 225-245, 1997.

[81] C. E. Close, M. R. Gleeson, and J. T. Sheridan, "Monomer diffusion rates in photopolymer material. Part I. Low spatial frequency holographic gratings," Journal of the Optical Society of America B, vol. 28, no. 4, pp. 658-666, 2011.

[82] C. E. Close, M. R. Gleeson, D. A. Mooney, and J. T. Sheridan, "Monomer diffusion rates in photopolymer material. Part II. High-frequency gratings and bulk diffusion," Journal of the Optical Society of America B, vol. 28, no. 4, pp. 842-850, 2011.

[83] D. Sabol, M. R. Gleeson, S. Liu, and J. T. Sheridan, "Photoinitiation study of Irgacure 784 in an epoxy resin photopolymer," Journal of Applied Physics, vol. 107, no. 5, Article ID 053113, 2010. 
[84] E. Hata, K. Mitsube, K. Momose, and Y. Tomita, "Holographic nanoparticle-polymer composites based on stepgrowth thiol-ene photopolymerization," Optical Materials Express, vol. 1, no. 2, pp. 207-222, 2011.

[85] E. Hata and Y. Tomita, "Stoichiometric thiol-to-ene ratio dependences of refractive index modulation and shrinkage of volume gratings recorded in photopolymerizable nanoparticle-polymer composites based on step-growth polymerization," Optical Materials Express, vol. 1, no. 6, pp. 1113-1120, 2011.

[86] R. A. Rupp, J. Hehmann, R. Matull, and K. Ibel, "Neutron diffraction from photoinduced gratings in a PMMA matrix," Physical Review Letters, vol. 64, no. 3, pp. 301-302, 1990.

[87] M. Fally, "The photo-neutronrefractive effect," Applied Physics B, vol. 75, no. 4-5, pp. 405-426, 2002.

[88] J. Klepp, C. Pruner, Y. Tomita et al., "Diffraction of slow neutrons by holographic $\mathrm{SiO}_{2}$ nanoparticle-polymer composite gratings," Physical Review A, vol. 84, no. 1, Article ID 013621, 2011.

[89] M. Fally, J. Klepp, and Y. Tomita, “An experimental studyto discriminate between the validity of diffraction theories for off-Bragg replay," Applied Physics B. In press.

[90] J. T. Sheridan, "A comparison of diffraction theories for ofd Bragg replay," Journal of Modern Optics, vol. 39, no. 8, pp. 1709-1718, 1992.

[91] P. Vayalamkuzhi, R. Joseph, K. Sreekumar, and C. S. Kartha, "Investigation on the panchromaticity of silver-doped poly (vinyl alcohol)/acrylamide photopolymer," Applied Optics, vol. 50, no. 18, pp. 2886-2891, 2011.

[92] S. Orlic, E. Dietz, S. Frohmann, and J. Rass, "Resolutionlimited optical recording in 3D," Optics Express, vol. 19, no. 17, pp. 16096-16105, 2011.

[93] D. A. Waldman, C. J. Butler, and D. H. Raguin, "CROP holographic storage media for optical data storage at greater than $100 \mathrm{bits} / \mu \mathrm{m}^{2}$," in Proceedings of the SPIE on Organic Holographic Materials and Applications, vol. 5216, pp. 10-25, August 2003.

[94] M. Ortuño, E. Fernández, S. Gallego, A. Beléndez, and I. Pascual, "New photopolymer holographic recording material with sustainable design," Optics Express, vol. 15, no. 19, pp. 12425-12435, 2007.

[95] S. Gallego, A. Marquez, M. Ortuno, S. Marini, I. Pascual, and A. Belendez, "Monomer diffusion in sustainable photopolymers for diffractive optics applications," Optical Materials, vol. 33, no. 11, pp. 1626-1629, 2011.

[96] S. Gallego, A. Marquez, M. Ortuno, S. Marini, and J. Frances, "High environmental compatibility photopolymers compared to PVA/AA based materials at zero spatial frequency limit," Optical Materials, vol. 33, no. 3, pp. 531-537, 2011.

[97] M. R. Gleeson, J. Guo, and J. T. Sheridan, "Optimisation of photopolymers for holographic applications using the Nonlocal photopolymerization driven diffusion model," Optics Express, vol. 19, no. 23, pp. 22423-22436, 2011.

[98] M. R. Gleeson, J. T. Sheridan, F. K. Bruder et al., "Comparison of a new self developing photopolymer with AA/PVA based photopolymer utilizing the NPDD model," Optics Express, vol. 19, no. 27, pp. 26325-26342, 2011.

[99] J. Guo, M. R. Gleeson, S. Liu, and J. T. Sheridan, "Non-local spatial frequency response of photopolymer materials containing chain transfer agents: part I. Theoretical modelling," Journal of Optics, vol. 13, no. 9, Article ID 095601, 2011.

[100] J. Guo, M. R. Gleeson, S. Liu, and J. T. Sheridan, "Non-local spatial frequency response of photopolymer materials containing chain transfer agents: part II. Experimental results," Journal of Optics, vol. 13, no. 9, Article ID 095602, 2011.
[101] M. R. Gleeson, J. Guo, and J. T. Sheridan, "Recent developments in non-local photopolymerisation driven diffusion model," in Proceedings of the SPIE, pp. 8429-8448, Brussels, Belgium, 2012. 

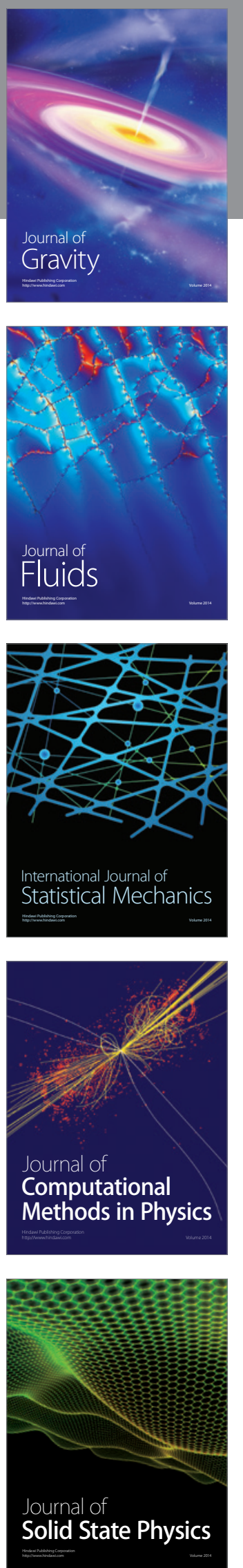

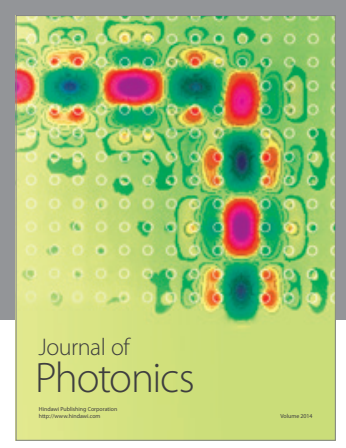

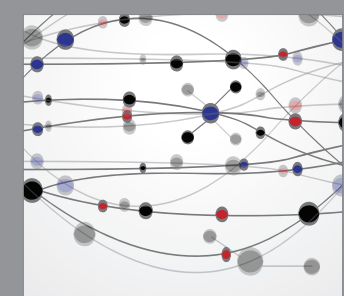

The Scientific World Journal
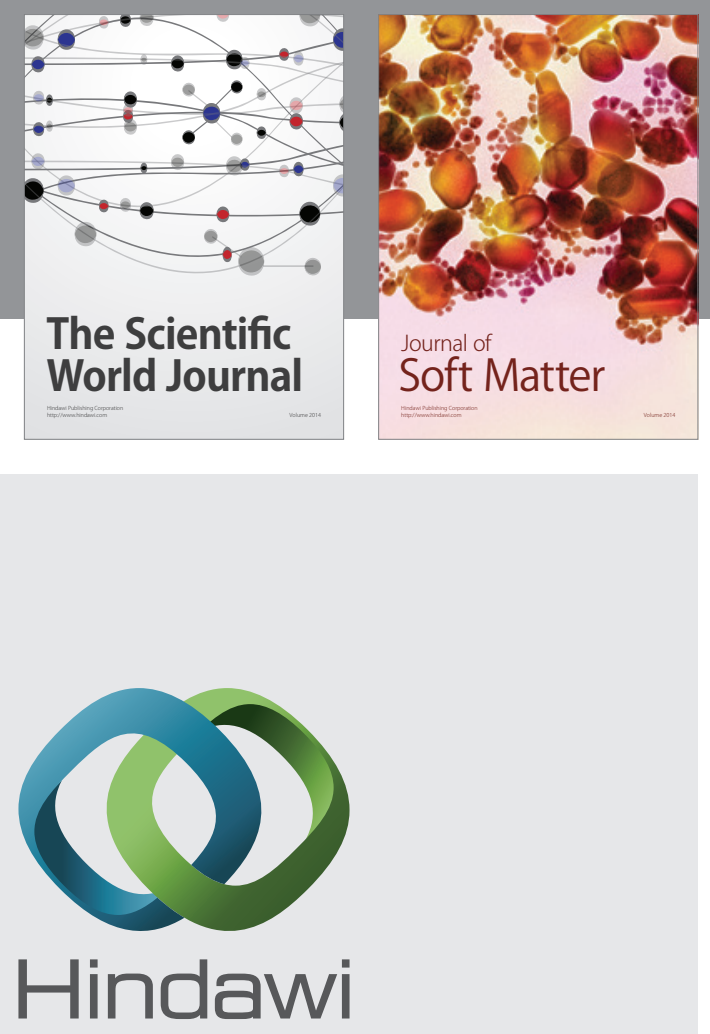

Submit your manuscripts at

http://www.hindawi.com
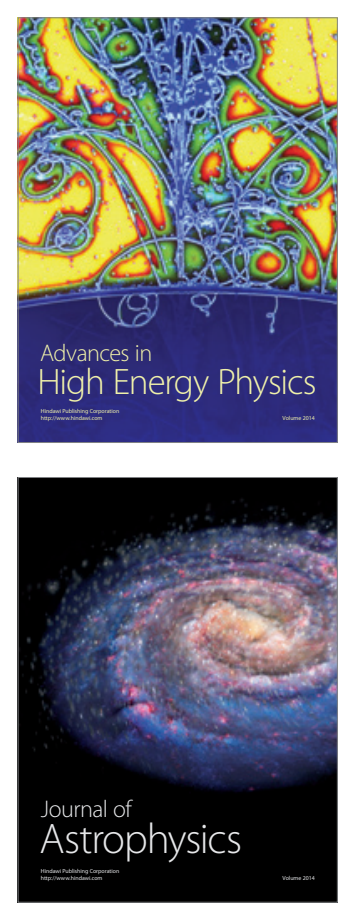
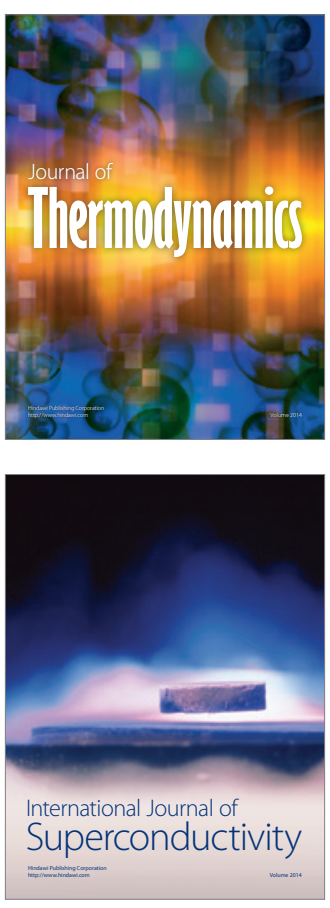
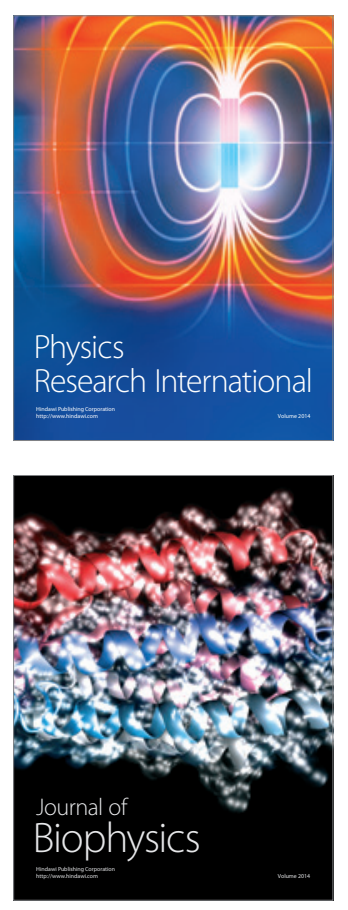
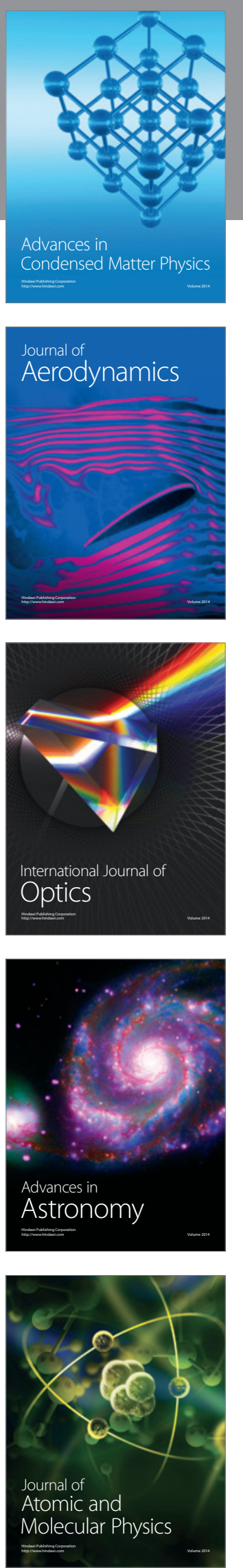